Arch. Math. 106 (2016), 3

(C) 2016 Springer International Publishing

0003-889X/16/010003-1

published online January 18, 2016

DOI 10.1007/s00013-016-0868-4

Archiv der Mathematik

\title{
Newly Appointed Editors
}

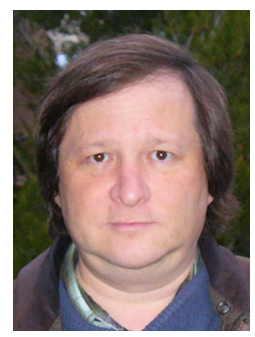

Alexander Borichev was educated at the University of St. Petersburg from 1980 to 1985 . From 1985 to 1992 he served as a Research Fellow at the Steklov Institute, St. Petersburg. He received there his doctoral degree in 1988. He was a Research Assistant at Uppsala University (1992-1996) and a Research Fellow at CNRS, Bordeaux (1996-2006). Since 2006 he is a Full Professor at the University of Aix-Marseille. His research is mainly devoted to Functional Analysis, Function Theory, Harmonic Analysis, and Approximation Theory.

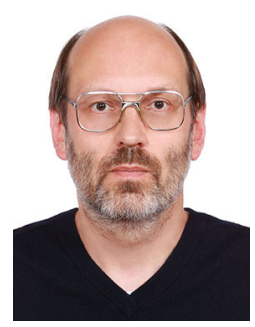

Andreas Schweizer was educated at Technische Universität Karlsruhe and Universität des Saarlandes, Saarbrücken, where he earned his doctoral degree in 1996. He held postdoctoral fellowships at CICMA, Montreal/Canada (1996-1999) and at Academia Sinica, Taipei/Taiwan (2000-2001). From 2001 to 2003, he served as a Research Fellow, and from 2003 to 2005, as Assistant Professor at KIAS, Seoul/Korea. After being lecturer at the University of Exeter/UK (2005-2008), he was a Visiting Associate Professor at NCTS, Hsinchu/Taiwan (2008-2010) and Assistant Research Fellow at Academia Sinica (2010-2012). Since 2012, he has been a Visiting Professor at KAIST in Daejeon/Korea. His research interest includes the arithmetic of function fields, arithmetic aspects of modular curves, Drinfeld modules and elliptic curves, linear groups over global fields and their integers, and value sharing of meromorphic functions (Nevanlinna theory).

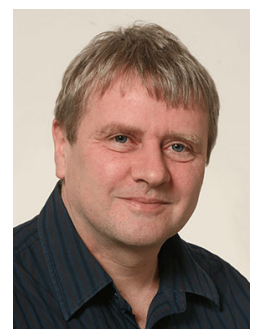

Roland Speicher (born 1960) studied mathematics and physics at the universities of Saarbrücken, Freiburg and Heidelberg. He received in Heidelberg his diploma degree (1986) in physics, and his PhD (1989) and his habilitation (1994) in mathematics. From 1995 to 2000 he was a Heisenberg Fellow of the DFG. From 2000 to 2010 he held a professorship at Queen's University in Kingston, Canada. Since 2010 he is Professor in Saarbrücken. He was awarded the Jeffery-Williams Prize of the Canadian Mathematical Society CMS in 2012 and an ERC Advanced Grant ("Non-commutative distributions in free probability") in 2014. He was an invited speaker at the ICM 2014 in Seoul. His research interests focus on free probability theory and random matrices; but include also, more generally, mathematical physics, operator algebras, and probability theory. 